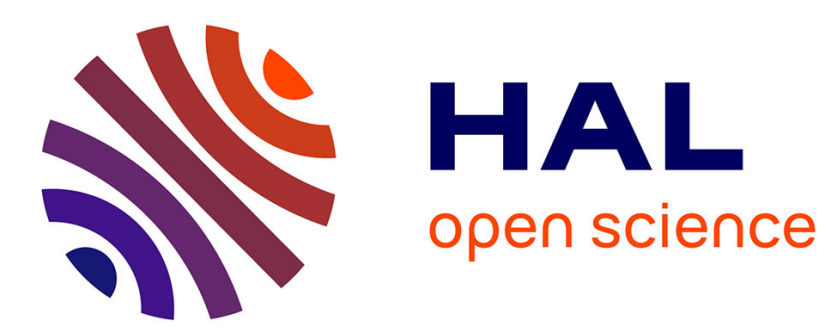

\title{
Multicommodity flow problems with a bounded number of paths : a flow deviation approach
}

\author{
Christophe Duhamel, Philippe Mahey
}

\section{To cite this version:}

Christophe Duhamel, Philippe Mahey. Multicommodity flow problems with a bounded number of paths: a flow deviation approach. Networks, 2007, Multicommodity Flows and Network Design, 49 (1), pp.80-89. 10.1002/net.20143 . hal-01691703

\section{HAL Id: hal-01691703 https://hal.science/hal-01691703}

Submitted on 24 Jan 2018

HAL is a multi-disciplinary open access archive for the deposit and dissemination of scientific research documents, whether they are published or not. The documents may come from teaching and research institutions in France or abroad, or from public or private research centers.
L'archive ouverte pluridisciplinaire HAL, est destinée au dépôt et à la diffusion de documents scientifiques de niveau recherche, publiés ou non, émanant des établissements d'enseignement et de recherche français ou étrangers, des laboratoires publics ou privés. 


\title{
Multicommodity flow problems with a bounded number of paths : a flow deviation approach
}

\author{
Christophe Duhamel, Philippe Mahey*
}

March 22, 2005

\begin{abstract}
We propose a modified version of the Flow Deviation method of Fratta, Gerla and Kleinrock to solve multicommodity problems with minimal congestion and a bounded number of active paths. We discuss the approximation of the min-max objective function by a separable convex potential function and give a mixed-integer non linear model for the constrained routing problem. A heuristic control of the path generation is then embedded in the original algorithm based on the concepts of cleaning the quasi inactive paths and the reduction of the fbw width for each commodity. Numerical experiments show the validity of the approach for realistic medium-size networks associated with routing problems in broadband communication networks with multiple protocols and label-switched paths (MPLS).
\end{abstract}

Keywords: Multicommodity fbw, routing, fbw deviation

\footnotetext{
*Laboratoire d'Informatique, de Modélisation et d'Optimisation des Systèmes, UMR 6158 CNRS, Université Blaise Pascal, Campus des Cézeaux, Aubière, France, email:\{christophe.duhamel,philippe.mahey\}@isima.fr
} 


\section{Introduction}

We will consider a network routing problem with multiple pairs of origins and destinations where we want to minimize the maximal relative congestion on the arcs of the network under the restriction that the number of paths used to carry the traffic is bounded. The problem of minimizing the fbw on the most congested link is of valuable interest for the design of data communication networks (see [4]). This basic problem is known to be hard to solve even if it can be written as a linear program. When additional constraints are present in the model, like path restrictions as considered here, it will result in very difficult problems for which exact methods are likely to be useless (see [15] for instance).

The particular path restriction we consider here is that the number of paths which support any feasible commodity fbw is bounded by a given number (possibly depending on the commodity). Of course, when that number is equal to 1 , we get the classical and difficult non bifurcated routing problem of unsplittable fbws as denoted by Kleinberg [13], and when the number is very large, we obtain the relatively easier routing problem with any fbw splitting allowed. The intermediate situation considered here is of practical interest when dealing with label switched paths (LSP) in modern broadband communication networks. Indeed, in multiple protocol labelswitched networks (MPLS) like all-optical IP backbones, different LSP are allowed to support the traffic for a given pair of nodes, but too many LSPs will deteriorate the performance of the protocols. One should thus try either to minimize the total number of LSPs (thus minimizing the number of wavelength conversions) with additional delay bounds to avoid congestion (see [3]), or to minimize the congestion with bounds on the number of supporting LSPs. Observe that the first choice turns to be much more intricated as it includes the problem of searching a minimal set of paths to carry a given feasible fbw, which is purely combinatorial. This is why we focus here on the second choice.

We will analyze here the adaptation of the Flow Deviation algorithm for Network Routing (see [4]) to this constrained minimal congestion problem. As the Flow Deviation method was designed to solve multicommodity fbw problems with separable convex costs, we will show first how the maximal congestion cost function can be approximated by these nonlinear functions, following earlier approaches based on polynomial approximation schemes and potential functions (see Bienstock's book for a comprehensive state-of-the art, [6]). 
The Flow Deviation will be briefly surveyed in section 3, with emphasis on the path fbw updates. In section 4 , the routing model with a bounded number of paths will be established and different heuristic procedures will be proposed. These algorithmic issues will be validated in the last section.

\section{Minimal congestion problems and potential func- tions}

In the remainder, we will call active a path carrying a positive path fbw.

Let $G=(V, E)$ be a directed graph such that $V$ is the set of nodes with $|V|=n$, and $E$ is the set of arcs with $|E|=m$. Each arc is assigned a capacity $C_{e}, e \in E$, and $T$ is a traffic requirement matrix such that, for each pair $(i, j)$ of nodes, $t_{i j}$ represents the amount of traffic required from node $i$ to node $j$. Each pair of nodes such that $t_{i j}>0$ will be referred to as a commodity and the index $k$ will be associated with a commodity, i.e. a pair of origin and destination nodes (respectively $o_{k}$ and $d_{k}$ ), and a traffic requirement $t_{k}=t_{o_{k}} d_{k}$. The total fbw on a given arc $e \in E$ will be denoted by $x_{e}=\sum_{k} x_{e}^{k}$ where $x_{e}^{k}$ is the amount of commodity $k$ routed on $\operatorname{arc} e$. In the nodearc formulation of the multicommodity fbw problem, we will need the node-arc incidence matrix $A$, (where $a_{i e}=+1, a_{j e}=-1$, if $e=(i, j) \in E$ ) to express the individual fbw constraints as $F_{k}=\left\{x^{k} \in \mathbb{R}^{m} \mid A x^{k}=b^{k}, x^{k} \geq 0\right\}$, where

$$
b_{i}^{k}= \begin{cases}+t_{k} & \text { if } i=o_{k} \\ -t_{k} & \text { if } i=d_{k} \\ 0 & \text { otherwise }\end{cases}
$$

The basic problem of minimizing the most congested arc in the routing of a multicommodity fbw consists in minimizing the piecewise linear convex function $f(x)=\max _{e} \frac{x_{e}}{c_{e}}$. The problem may nevertheless be modelled as a linear program by adding an additional variable $z$ as described below :

$$
\begin{aligned}
(M I N C O N G) & \min \quad z \\
\text { s.t. } & \begin{cases}\sum_{k} x_{e}^{k}-C_{e} z \leq 0, & \forall e \in E \\
x^{k} \in F_{k}, & k=1, \ldots, K\end{cases}
\end{aligned}
$$


Even if it is a linear multicommodity fbw problem, thus an LP which can be solved by standard decomposition techniques exploiting the underlying fbw structure, it is generally considered a hard problem. The first reason why this occurs is that the objective function $f$ is convex piecewise linear and not separable with respect to arcs. The second reason is that it produces optimal solutions with a large number of active paths, being in that sense equivalent to the Maximum Concurrent Flow problem as shown below. Bienstock has reported in [6] a set of numerical experiments on very large maximum concurrent fbw problems (with up to $4 \bullet 10^{5}$ rows and $2 \bullet 10^{6}$ columns) exhibiting abnormal cubic growth of the cpu time to solve them with the CPLEX dual code.

Observe that (MINCONG) is formulated without explicit capacity constraints on the total arc fbws. This means that, besides its natural applications to congestion control in data networks, the routing problem (MINCONG) may be considered as an optimization formulation of the multicommodity fbw feasibility problem, as we have the following relations : (MINCONG) is feasible if and only if there exists at least one path linking each origin to its destinations. Let $z^{*}$ be an optimal value for (MINCONG); there exists a feasible multicommodity fbw if and only if $z^{*} \leq 1$.

The path structure of an optimal solution of (MINCONG) can be analyzed by considering its arc-path formulation. Let $P_{k}$ be the set of paths linking origin $o_{k}$ with destination $d_{k}$ and $x_{k p}$ be the path fbw fbwing on path $p \in P_{k}$, i.e. $x_{k p}$ is the portion of the demand $t_{k}$ routed on path $p$. The arc-path version of (MINCONG) is then :

$$
\begin{aligned}
& (P C O N G) \quad \min \quad z \\
& \text { s.t. }\left\{\begin{array}{lll}
\sum_{k} \sum_{p ; e \in p} x_{k p}-C_{e} z & \leq 0 & e \in E \\
\sum_{p \in P_{k}} x_{k p} & =t_{k} & k=1, \ldots, K \\
x_{k p} & \geq 0 & k=1, \ldots, K, p \in P_{k}
\end{array}\right.
\end{aligned}
$$

We recall that a path is active when it carries some positive fbw; an arc $e$ will be called critical when it corresponds to the maximal congestion, i.e. when $x_{e}=C_{e} z$. An active path containing critical edges will be called a critical path. Suppose now that some commodity is routed on a critical path $p$ at the optimal solution and that there exists a second active path $p^{\prime}$ supporting that commodity which is not critical. Both paths define a cycle, so that one can modify the solution deviating a small quantity from $p$ to $p^{\prime}$. A new basic optimal solution should be obtained when $p^{\prime}$ 
turns to be critical. That situation can thus only occur when there are multiple optimal solutions, else :

Proposition 1 Suppose (PCONG) has a unique optimal solution; then, if any commodity is routed on a critical path in an optimal solution of (PCONG), then all paths used to route that commodity are critical.

An optimal solution to (PCONG) will contain at most $K+\sigma$ active paths (and at least $K$ ), where $\sigma$ is the number of critical edges.

As observed in [21], (MINCONG) is also strongly related to the Maximum Concurrent Flow problem (MAXTHRU), where one wants to maximize the throughput of the network for a given set of capacities. The throughput $Z$ of the network is a load factor that multiplies the traffic matrix. In the model shown below, $X^{k}$ represents again the $k$-th commodity fbw :

$$
\begin{aligned}
\max Z \\
\text { s.t. }\left\{\begin{array}{l}
\sum_{k} X_{e}^{k} \leq C_{e} \\
X^{k}
\end{array} \in F_{k}(Z) \quad k=1, \ldots, K\right.
\end{aligned}
$$

where $F_{k}(Z)=\left\{X^{k} \in \mathbb{R}^{m} \mid A X^{k}=Z b^{k}, X^{k} \geq 0\right\}$.

One can easily verify that, for any optimal solution $x^{*}$ of (MINCONG), with optimal value $z^{*}$, there exists an optimal solution $X^{*}$ of (MAXTHRU), with optimal value $Z^{*}$, such that $x^{*}=z^{*} X^{*}$ and $z^{*}=1 / Z^{*}$. Moreover, the crucial fact is that both problem solutions share the same set of active paths.

Both problems (MINCONG) and (MAXTHRU) have received a lot of attention in the past decade, namely since the seminal paper by Shahrokhi and Matula [21] who first proposed a fully polynomial approximation scheme to solve (MAXTHRU) with uniform capacities. They showed that the minimization of a separable exponential penalty function on the arcs yields a fbw with a nearly maximal throughput. They chose the following penalty function :

$$
\phi_{e}\left(x_{e}\right)=\exp \left(\frac{2 m^{2}}{\varepsilon} x_{e}\right)
$$


where $\varepsilon$ is a positive parameter which defines the approximation. They found a complexity bound of $O\left(\mathrm{~nm}^{7} / \varepsilon^{5}\right)$ and showed that the number of active paths is in $O\left(m^{3} / \varepsilon^{2}\right)$. Faster algorithms based on refinements of that exponential penalty function have been proposed later and extended to non uniform capacities and to other packing and covering problems (see [17], [12], [11]). Following Bienstock in [6], we will denote these separable penalty functions by the term of potential functions.

\section{The flow deviation method}

The Flow Deviation method (FD) is an adaptation of the classical linearization algorithm of Frank and Wolfe [9] to solve multicommodity fbw problems with convex costs. It has been first proposed by Fratta, Gerla and Kleinrock [10] in the context of designing packet-switched networks and has been widely used by the transportation community (see [16]) and in telecommunications networks (see [4]). We recall below the formal ideas behind Frank-Wolfe's algorithm for general nonlinear programs with linear constraints :

$$
\begin{array}{rc}
\text { Minimize } & \Phi(x) \\
\text { s.t. } & A x=b \\
& x \geq 0
\end{array}
$$

The method proceeds by successive linearization solving LP subproblems at each iteration $t$ where the gradient $\nabla \Phi\left(x^{t}\right)$ has been computed. Let $\tilde{x}^{t}$ be the optimal solution of the subproblem at iteration $t$

$$
\tilde{x}^{t}=\operatorname{Argmin}_{x \in P} \nabla \Phi\left(x^{t}\right) \bullet x
$$

where $P=\left\{x \in \mathbb{R}^{n} \mid A x=b, x \geq 0\right\}$ is the polyhedron of feasible solutions supposed bounded. Thus, we can assume that $\tilde{x}^{t}$ is an extreme point of $P$. The direction $d^{t}=$ $\tilde{x}^{t}-x^{t}$ is a descent direction in the sense that the directional derivative $\nabla \Phi\left(x^{t}\right) \bullet d^{t}$ is strictly negative. Then the new iterate is obtained by carrying out a line search on the segment $\left[x^{t}, \tilde{x}^{t}\right]$ with the non linear function $\Phi$. Convergence results have been obtained in the strictly convex case (see [5] for example) but the method suffers from very slow convergence tail which is evidenced by the following fact : the solution of the non linear program is in general not a vertex of the feasible polyhedron so 
that, as the sequence of feasible solutions converges towards a point on a face of the polyhedron, the angle between successive descent directions tends to $180^{\circ}$ (see Fig. 1). It is shown in [5] how typical sublinear convergence rate can be exhibited in very simple and low-dimensional situations. This drawback is somehow compensated by the observed fact that nearly optimal solutions -typically within $1 \%$ of optimality can be obtained very quickly.

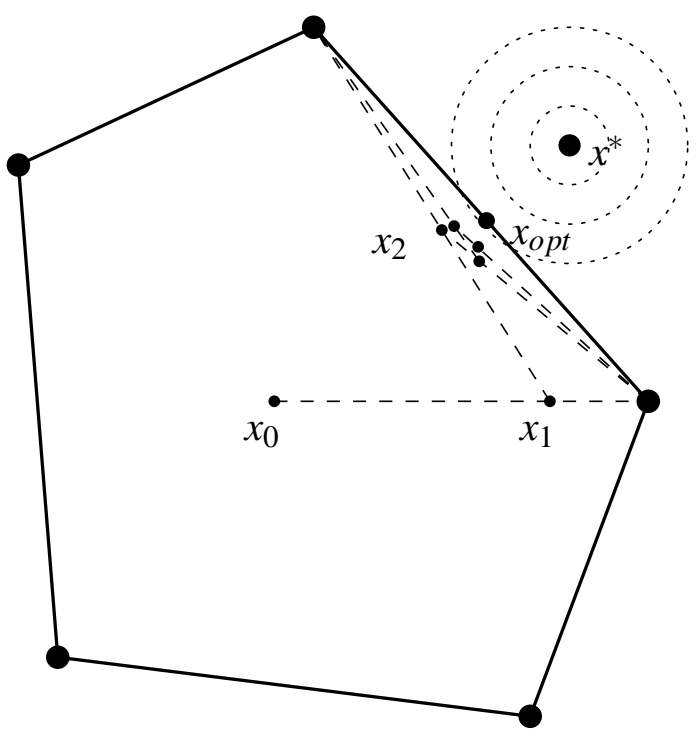

Figure 1: Zigzagging convergence of Frank and Wolfe's method

On the other hand, the positive aspect of the implementation of Frank-Wolfe's method to multicommodity fbw problems is the simplicity of the subproblems resolutions which reduce to shortest-path computation and the solution update which consists in fairly deviating fbws from the active paths towards the new ones without explicitly computing the individual commodity fbws as we can see below.

Now, to apply the Flow Deviation method to (MINCONG), we need to approximate the non smooth congestion function $f(x)=\max _{e} x_{e} / C_{e}$ by a smooth convex function. This can be done by using a separable potential function as shown in the former section. An interesting link between the Flow Deviation algorithm and the resolution of (MINCONG) has been analyzed recently by Bienstock and Raskina in [7]. They proposed an algorithm to solve (MINCONG)which alternates between a magnification step where the throughput is increased for a fixed congestion and 
a potential reduction step where congestion is minimized for a fixed throughput. That latter step is realized by performing fbw deviation inner steps with the Kleinrock's congestion function used in [10]. The interesting fact is that this function has an interpretation as a measure of the Quality of Service (QoS) of the network. It is indeed the average delay suffered by a data packet on arc $e$. In [10], independence assumptions with Poisson arrivals on the queuing network lead to Kleinrock's delay function which is proportional to $\Phi_{e}\left(x_{e}\right)=\frac{x_{e}}{C_{e}-x_{e}}$. The total cost is simply $\Phi(x)=\sum_{e} \Phi_{e}\left(x_{e}\right)$. Observe that the objective function acts as a barrier function on the capacity constraints which can thus be ignored in the model :

$$
\begin{array}{rc}
\text { Minimize } & \Phi(x) \\
\text { s.t. } & x^{k} \in F_{k}, k=1, \ldots, K
\end{array}
$$

where $F_{k}=\left\{x^{k} \in \mathbb{R}^{|E|} \mid A x^{k}=b^{k}, x^{k} \geq 0\right\}$ is the set of fbws for commodity $k$.

Assume that we get a strictly feasible multicommodity fbw at iteration $k$, i.e. such that $x_{e}<C_{e}, \forall e$. Then, the linearization step of (FD) reduces to separate shortest-path computations for each commodity $k$ with arc lengths $\nabla \Phi_{e}\left(x_{e}^{t}\right)$. The solution $\tilde{x}^{t}$ corresponds to the situation where all demands $t_{k}$ are routed separately on these new paths. Note that $\tilde{x}^{t}$ may not satisfy the capacity constraints. The new solution $x^{t+1}$ is obtained by carrying out a line search on the segment $\left[x^{t}, \tilde{x}^{t}\right]$. Note again that the new solution will be forced to strict feasibility by the barrier function.

The main point in the procedure above is the fact that all computations are performed on the total fbw variables. However, in some situations, one may be interested in computing the optimal path fbws. An arc-path formulation is then necessary and the (FD) iterations can be carried out on the path fbw variables as explained in [4]. The first step of the procedure is unchanged so that, if $\tilde{p}$ is the shortest path with the first-derivative arc lengths for a given commodity $k$, then we set $\tilde{x}_{k} \tilde{x}_{p}=t_{k}$ and $\tilde{x}_{k p}=0$ for $p \neq \tilde{p}$, so that the fbw deviation step can be computed in the following way :

$$
x_{k p}^{t+1}=x_{k p}^{t}+\theta_{t}\left(\tilde{x}_{k p}^{t}-x_{k p}^{t}\right), \forall p \in P_{k}
$$

where $\theta_{t}$ is the optimal step size which minimizes the objective function over all $\theta \in[0,1]$. As mentioned before, the method is a descent algorithm so that the values $\Phi\left(x^{t}\right)$ decrease monotonically. On the other hand, we can obtain easily a lower bound of the optimal value at each iteration by computing $\tilde{L B}_{t}=\Phi\left(x^{t}\right)+\nabla \Phi\left(x^{t}\right) \bullet$ 
$\left(\tilde{x}^{t}-x^{t}\right)$; these succesive values need not increase monotonically, so we must keep the highest value as the current lower bound

$$
L B_{t}=\max _{h=1, \ldots, t} \tilde{L B_{h}}
$$

In the original (FD) method, an equal fraction of the fbw on the nonshortest paths is shifted to the shortest path. One may observe that this update of path fbws will tend to increase monotonically the number of active paths. Variants of the basic Flow Deviation method can be built by modifying one of the inner steps of the algorithm, i.e.

- either by modifying the search direction, for instance by substituting the shortestpath calculations by min-cost fbw subproblems for each commodity,

- or by deviating non uniform fbw proportions on the newly generated paths.

To understand the first strategy, one can add the redundant constraints $x_{e}^{k} \leq C_{e}, \forall e, k$ to the model (MINCONG); after the linearization, the direction-finding subproblem of (FD) decomposes now in $K$ minimum-cost fbw problems. The computational overhead of these subproblems compared to the original shortest-path calculations is compensated by the choice of feasible paths (considering one commodity at a time). But the drawback is that more paths are likely to be generated which is exactly what we do not wish, and the path fbw updates are not so straightforward as in the original method. Our testing with that variant has confirmed these difficulties and we had to decide not to implement it in our algorithm. Observe nevertheless that some authors have chosen to implement min-cost fbw computations instead of shortest-path calculations to improve the worst-case behaviour of some approximation algorithms (see [12] or [20]).

There are many different strategies to modify the search direction which result in non uniform deviations from the current active paths to the shortest one. Secondorder information can be used to yield Newton or Quasi-Newton directions as suggested in [4]. Conjugate gradient strategies have also been tested in earlier works (see [14]). Again, the expected gain in convergence rate is overtaken by the extra work of performing each iteration and reconstituting the path support.

We will discuss in the last section heuristic procedures to carry out (FD) steps with a bounded number of paths. 


\section{Bounding the number of paths}

We will finally consider a more complex but realistic situation where some path constraints are added to the (MINCONG) model. A way to find an interesting compromise between low congestion and unsplittable fbw is to add some constraints on the number of active paths for each commodity. The general case has been scarcely considered in the literature, the research focussing mainly on the very special cases where either a single path is forced for each commodity or two disjoint paths are required for security reasons (see Kleinberg's thesis [13] for example). Another interesting type of constraints is the case of $k$-splittable fbws where each commodity fbw may be splitted uniformly among $k$ routes [2]. Besides these studies, approximation and heuristic techniques have been devoted to the reduction of the number of supporting paths with a linear or non linear objective function (see [8], [18], [19]).

Let $R_{k}, k=1, \ldots, K$ be the maximum number of paths on which commodity $k$ can be routed. Thus a feasible routing is a set of $R_{k}$ paths chosen in the set $P_{k}$ for each $k$ such that $\sum_{p=1}^{R_{k}} x_{k p}=t_{k}, k=1, \ldots, K$. It can be referred to as a (non uniform) $k$-splittable fbw, following Skutella and others. In [18], additional constraints on the size of the trunks (path capacities) are considered. We will not consider these constraints here but instead use the congestion objective function discussed in the previous sections. These problems are all known to be NP-complete as soon as $R_{k}$ is lower than the number of paths supporting the optimal solution of (PCONG). A mixed-integer programming formulation can be formalized to model the bound on the number of paths, introducing 0-1 variables associated with each path in (PCONG) :

$(B P C O N G) \quad \min z$
s.t. $\left\{\begin{array}{lll}\sum_{k} \sum_{p ; e \in p} x_{k p}-C_{e} z & \leq 0 \quad e \in E \\ \sum_{p \in P_{k}} x_{k p} & =t_{k} \quad k \in \mathcal{K} \\ x_{k p}-t_{k} y_{k p} & \leq 0 \quad k \in \mathcal{K}, p \in P_{k} \\ \sum_{p \in P_{k}} y_{k p} & \leq R_{k} \quad k \in \mathcal{K} \\ x_{k p} & \geq 0 \quad k \in \mathcal{K}, p \in P_{k} \\ y_{k p} & \in\{0,1\} \quad k \in \mathcal{K}, p \in P_{k}\end{array}\right.$

In the following, the number of active paths to support a given feasible fbw will be 
called the flow width. If one solves a capacitated multicommodity fbw problem with linear costs, the total number of active paths at the solution is at most $K+m$, and, supposing $K=O(m)$, this results in an average of 2 active paths per commodity. In practice, unfortunately, many commodities will be routed on a single path and a few of them will spread their fbw on a large number of paths. Now considering an uncapacitated model with a nonlinear barrier function as above, the fbw width at the optimal solution can be bounded thanks to Caratheodory's theorem by $m+1$ for each commodity. This is still a too large number and, even worse, the behaviour of the (FD) procedure will tend to add more paths than necessary in the construction of the current solution. Indeed, supposing one new path is generated at each iteration, as the procedure deviates fbw in equal proportion from all active paths to these new ones, the fbw width will monotonically increase with the iteration count. This nasty fact could be avoided at the cost of eliminating redundant paths in the definition of the current solution as a convex combination of path fbws. Typically, pivoting steps are needed to compute the smallest representation in term of path fbws.

The question we address here is how to maintain a limited number $R$ of paths in the (FD) process. The difficulties to achieve this goal are :

- Global optimality will in general be far out of reach because most situations will result in NP-hard problems;

- the arc-path model being implicit, we can only reroute the fbws issued from the cancelled paths towards the already generated paths;

- feasibility issues turn to be crucial as one does not know if the network will be able to support the traffic on a smaller number of paths.

The central idea to approximately solve (BPCONG) is to adapt the Flow Deviation (FD) to the case of $k$-splittable fbws. One can observe that the same idea has been used very early in the seminal paper by Fratta, Gerla and Kleinrock [10] who applied it to the particular case of unsplittable fbws $\left(R_{k}=1, \forall k\right)$. The heuristic procedure simply tries to deviate all the demand on the newly generated shortest paths without violating any capacity constraint. This can be performed commodity per commodity until no improvement in the objective function is observed. It seems clear that that simple method can stop too early with a very poor feasible solution unless the traffic load is very low. Besides that, the major problem with the adaptation of (FD) to $k$-splittable fbws is the accumulation of active paths in the iterative 
process. Indeed, the fbw is deviated in equal proportions from all current paths to load the new one and, either all previous path fbws are set to zero $\left(\theta_{t}=1\right)$ or all are decreased $\left(0<\theta_{t}<1\right)$ and the fbw width increased by one unit, the latter situation being the most likely to occur. Now observe that the general problem to find a set of paths supporting a given feasible fbw has in general many solutions. Indeed, if $M_{k}$ is the arc-path incidence matrix associated with the set $P_{k}^{t+1}$ of paths for the $k$-th commodity after updating the fbws at iteration $t$ and $x^{t+1}$ (resp. $f^{t+1}$ ) is the path fbw vector for the new active paths (resp. the arc fbw vector), we look for a solution of the following linear system :

$$
\left[\begin{array}{ccccc}
M_{1} & \cdots & M_{k} & \cdots & M_{K} \\
e^{T} & & & & \\
& \ddots & & & \\
& & e^{T} & & \\
& & & \ddots & \\
& & & & e^{T}
\end{array}\right] x^{t+1}=\left[\begin{array}{c}
f^{t+1} \\
d_{1} \\
\vdots \\
d_{k} \\
\vdots \\
d_{K}
\end{array}\right]
$$

where $e$ is a vector of 1 . This system always possess at least one solution with non negative components (the one which corresponds to the classical uniform deviation and used all paths in the current representation), but we should be able to find a better solution using less paths for the same arc fbw values. Unfortunately, the problem to find a minimum supporting path set for a given fbw, i.e. to compute the minimal fbw width, is NP-hard (see [22]). Moreover, the indetermination in the definition of the path support is more intricate in the case of multicommodity fbws as, for a given feasible multicommodity fbw, there are in general an infinity of individual arc fbw solutions which satisfy the multicommodity fbw constraints $\sum_{k} x_{e}^{k}=x_{e}, \forall e \in E$. For a single positive fbw on a directed graph, the fbw decomposition theorem (see [1]) guarantees there exists a support with at most $n+m$ paths and cycles (and at most $m$ cycles). When the only directed cycles in the network are the ones which use the return arc from the sink back to the source, it can be easily seen that the minimum number of active paths is bounded above by $m-n+2$ (using paths with linearly independent incidence vectors). But this number will in general be too large to be of practical interest as network protocols do not allow for a splitting in more than, say, 10 paths, even in large dense networks.

The basic ingredients of our procedure are :

Cleaning the poorest routes: the cleaning procedure is based on the idea that some routes belonging to the initial solution may not belong to the optimal solution. Then, 
the fbw deviation procedure will decrease the amount of fbw but will still maintain a positive quantity of fbw on these routes. Thus a first strategy to reduce the number of paths consists in canceling the paths which support a quantity of fbw which is less than a given relative threshold. The solution is then updated, either by optimizing the cost on the corresponding reduced set of paths before generating any new path, or by fairly sharing the canceled quantity of traffic on the remaining paths. Observe that the new objective function value can have increased during that phase, but in most situations, the canceled paths will not be active any more in the future solutions, including the optimal one.

Iterative loading of commodities: a second strategy, already mentioned in the case of unsplittable fbws, consists in updating the fbw one commodity at a time. This can be interpreted as a Gauss-Seidel like version of the Flow Deviation method, where the line-search is performed on a single commodity fbw. As a consequence, the stepsize $\theta$ wil be greater and more path fbws will decrease significantly with a higher probability to be canceled.

Avoiding unprofitable shortest path calculations: in the original (FD) algorithm, a shortest path is computed for each commodity at each iteration. However, computational experiments show that, most of the time, the new shortest path already belongs to the current solution set of active paths. Only few brand new improving paths are identified during the optimization (typically, less than 100 paths are stored at the end of several thousands FD iterations). Then, it may be interesting to try to look for an improving path among the active paths instead of computing a shortest path. We have modified the improving path selection in the following way : during a given number $\tau$ of iterations, the improving path is computed among the active paths. At the begining, $\tau \leftarrow 10$. Then, at the end of the minor iterations, a shortest path is computed. If this path is also an active one, then $\tau \leftarrow 2 \tau$ otherwise $\tau \leftarrow 10$.

Controlling the flow width: there are two ways to control the fbw width for each commodity. The first one (external) is based on relaxing first the width constraint, then trying to satisfy it progressively. The second one (internal) keeps the width constraint during the fbw deviation procedure. In the external procedure, the width constraint is restored using the same idea as in the cleaning procedure. At each iteration of the restoration, the active path with the smallest amount of fbw is fairly rerouted among the remaining ones, then a fbw deviation with $\tau \leftarrow \infty$ is applied to locally optimize the fbw structure. A path control is added at each step in the internal procedure : once a fbw reaches its width limit, $\tau \leftarrow \infty$, if its width drops 
below the limit, then $\tau \leftarrow 10$.

\section{Numerical experiments}

The (FD) algorithm and its variants adapted to problem (BPCONG) have been coded in $\mathrm{C}$ and compiled through gec 3.2 on the gnu/linux system. The numerical tests were performed on an Intel P3 $800 \mathrm{MHz}$ computer with $256 \mathrm{Mb}$ RAM.

Test networks: The test networks used for the numerical experimentation are of two types : 1) a realistic core network instance with 12 nodes, 88 arcs and 74 commodities; 2) a testbed of 20 random instances; those instances are built on planar graphs (obtained through Delaunay triangulation) whose size ranges from 10 to 40 nodes. For each size, 5 different sets of demands have been randomly defined. The number of commodities has been set to $2 n, 4 n, 6 n, n(n-1) / 2$ and $n(n-1)$ where $n$ is the number of nodes.

Initialization procedure: fbw deviation needs an initial solution. Thus, one has to provide a way to compute a feasible solution. This is achieved through the following heuristic, denoted by INITFD. It first consists in cutting all demands into equal-sized packets. Then each packet is routed, one at a time, on the shortest path where arc costs are the load first derivative. Arc loads are updated after each packet has been sent. Packets are randomly chosen from the remaining ones at each iteration to prevent bias and ill behaviour. However, this is not sufficient to guaranty feasibility of the solution since wrong routing decisions may be made. In practice, when the network is not close to saturation, those problems are not likely to appear.

In Table 1, results are shown for varying values of the throughput $\gamma$ (a common multiplying factor for all commodities demand). INITFD, FD and FD+ rows respectively give informations for the heuristic, the classical fbw deviation method and the fbw deviation method using the cleaning procedure and the improved path generation. For each method and each $\gamma$, CPU time in seconds, value of the optimal solution are reported. The last seven columns give the number of commodities in the solution with the given number of active paths. The cleaning procedure (FD+ method) helps a lot reducing the number of active paths. Combined with the modified path generation, they also provide a strong reduction in CPU time. The reason is that the cleaning procedure removes paths that are not likely to be in the optimal 


\begin{tabular}{llrrrrrrrrr}
\hline$\gamma$ & meth. & time $(\mathrm{s})$ & $z^{*}$ & $\# 1$ & $\# 2$ & $\# 3$ & $\# 4$ & $\# 5$ & $\# 6$ & $\# 7$ \\
\hline \multirow{3}{*}{0.5} & INITFD & 9.23 & 9.295 & 44 & 14 & 8 & 8 & 0 & 0 & 0 \\
& FD & 4.36 & 9.169 & 43 & 13 & 10 & 8 & 0 & 0 & 0 \\
& FD+ & 0.07 & 9.169 & 46 & 15 & 13 & 0 & 0 & 0 & 0 \\
\hline & INITFD & 9.64 & 28.014 & 39 & 16 & 10 & 5 & 0 & 2 & 2 \\
1.0 & FD & 84.63 & 27.418 & 38 & 14 & 10 & 8 & 0 & 0 & 4 \\
& FD+ & 5.01 & 27.418 & 38 & 23 & 11 & 2 & 0 & 0 & 0 \\
\hline & INITFD & 10.71 & 73.739 & 39 & 18 & 8 & 5 & 0 & 2 & 2 \\
1.5 & FD & 372.68 & 71.186 & 38 & 14 & 10 & 8 & 0 & 0 & 4 \\
& FD+ & 43.01 & 71.186 & 40 & 20 & 11 & 1 & 2 & 0 & 0 \\
\hline
\end{tabular}

Table 1: cleaning procedure and improved path generation ( $\gamma$ influence)

solution. Thus, removing those suboptimal paths avoid a lot of iterations that would otherwise be needed to reduce their amount of fbw down to zero.

Table 2 shows the impact of the required final gap (column "gap") on the FD and FD+ methods. The meaning of the columns is the same as before. It can be clearly seen that the cleaning procedure and the internal path generation help keeping low CPU time as well as providing solutions with a low number of path per commodity. This also suggests the fact that FD+ as a better experimental convergency towards the optimal solution.

Table 3 summarizes the behaviour of FD and FD+ for the whole set of instances. Again, FD+ compares well against FD. BLABLABLA `a ajouter

Table 4 illustrates the path control procedure on the optimal solution. The first column shows the width upper bound $R$ for every commodities. In all cases, fbw 


\begin{tabular}{clrrrrrrrrr}
\hline gap & meth. & time $(\mathrm{s})$ & $z^{*}$ & $\# 1$ & $\# 2$ & $\# 3$ & \#4 & \#5 & \#6 & \#7 \\
\hline- & INITFD & 9.64 & 28.014 & 39 & 16 & 10 & 5 & 0 & 2 & 2 \\
\hline $10^{-1}$ & FD & 0.00 & 28.012 & 38 & 17 & 10 & 5 & 0 & 2 & 2 \\
& FD+ & 0.00 & 28.012 & 38 & 17 & 10 & 5 & 2 & 1 & 1 \\
\hline $10^{-5}$ & FD & 0.06 & 27.629 & 38 & 14 & 10 & 8 & 0 & 2 & 2 \\
& FD+ & 0.03 & 27.629 & 38 & 15 & 11 & 6 & 2 & 2 & 0 \\
\hline $10^{-3}$ & FD & 0.95 & 27.441 & 38 & 14 & 10 & 8 & 0 & 0 & 4 \\
& FD+ & 0.50 & 27.441 & 38 & 14 & 18 & 4 & 0 & 0 & 0 \\
\hline $10^{-4}$ & FD & 17.61 & 27.420 & 38 & 14 & 10 & 8 & 0 & 0 & 4 \\
& FD+ & 4.73 & 27.418 & 38 & 22 & 12 & 2 & 0 & 0 & 0 \\
\hline $10^{-5}$ & FD & 84.63 & 27.418 & 38 & 14 & 10 & 8 & 0 & 0 & 4 \\
& FD+ & 5.01 & 27.418 & 38 & 23 & 11 & 2 & 0 & 0 & 0 \\
\hline
\end{tabular}

Table 2: cleaning procedure and internal path generation (gap influence)

deviation with internal path management (FD+int) requires less CPU time than fbw deviation with external path management (FD+ext). There are two reasons: first, when FD+int reaches some commodity width limit, it is quite difficult to find an alternate path to exchange with an active one. Second, removing an active path in FD+ext does a lot of perturbation. The quality of the solution for both methods is nearly the same. No accurate gap can be produced since the only valid lower bound is unconstrained fbw deviation lower bound.

Finaly, the last table shows fbw deviation behaviour for the unsplittable case that is, when $R=1$. We compare fbw deviation with internal path management (FD+int) against two heuristics. H1 (resp. H2) sorts the commodities by decresing (resp. increasing) demands and then route each one on the shortest path, where the cost are the delay first derivative. Those costs are updated after each routing. It can be seen $\mathrm{H} 1$ is quite close to FD for a much lesser CPU time. This shows the limits of our approach, when the width constraint is very low. 


\begin{tabular}{|c|c|c|c|c|}
\hline problem & $\mathrm{n}$ & $k$ & $\begin{array}{ll} & \mathrm{FD} \\
z^{*} & \text { time (s) }\end{array}$ & $\begin{array}{ll} & \text { FD+ } \\
z^{*} & \text { time (s) }\end{array}$ \\
\hline$T \_10 \_20$ & 10 & 20 & & \\
\hline$T \_10 \_40$ & 10 & 40 & & \\
\hline$T \_10 \_45$ & 10 & 45 & & \\
\hline$T \_10 \_60$ & 10 & 60 & & \\
\hline T_10_90 & 10 & 90 & & \\
\hline$T \_20 \_40$ & 20 & 40 & & \\
\hline$T \_20 \_80$ & 20 & 80 & & \\
\hline$T \_20 \_120$ & 20 & 120 & & \\
\hline$T \_20 \_190$ & 20 & 190 & & \\
\hline$T \_20 \_380$ & 20 & 380 & & \\
\hline$T \_30 \_60$ & 30 & 60 & & \\
\hline$T \_30 \_120$ & 30 & 120 & & \\
\hline$T \_30 \_180$ & 30 & 180 & & \\
\hline$T \_30 \_435$ & 30 & 435 & & \\
\hline$T \_30 \_870$ & 30 & 870 & & \\
\hline$T \_40 \_80$ & 40 & 80 & & \\
\hline$T \_40 \_160$ & 40 & 160 & & \\
\hline$T \_40 \_240$ & 40 & 240 & & \\
\hline$T \_40 \_780$ & 40 & 780 & & \\
\hline$T \_40 \_1560$ & 40 & 1560 & & \\
\hline
\end{tabular}

Table 3: results for the random instances

\begin{tabular}{clrrrrrr}
\hline$R$ & meth. & time (s) & $z^{*}$ & $\# 1$ & $\# 2$ & $\# 3$ & $\# 4$ \\
\hline no & FD & 5.03 & 27.418 & 38 & 23 & 11 & 2 \\
\hline 3 & FD+int & 1.90 & 27.438 & 38 & 26 & 10 & 0 \\
& FD+ext & 8.53 & 27.418 & 38 & 29 & 7 & 0 \\
\hline 2 & FD+int & 9.47 & 27.478 & 40 & 34 & 0 & 0 \\
& FD+ext & 33.38 & 27.478 & 39 & 35 & 0 & 0 \\
\hline 1 & FD+int & 12.05 & 39.608 & 74 & 0 & 0 & 0 \\
& FD+ext & 15.41 & 39.608 & 74 & 0 & 0 & 0 \\
\hline
\end{tabular}

Table 4: path control 


\begin{tabular}{crrrr}
\hline \multicolumn{3}{c}{ starting solution } & \multicolumn{2}{c}{ integer fbw FD } \\
heur. & value & time (s) & value & time (s) \\
\hline H1 & 39.614 & 0.00 & 39.577 & 0.00 \\
H2 & 175.509 & 0.00 & 39.575 & 0.01 \\
\hline & & FD+int & 39.608 & 12.05 \\
\hline
\end{tabular}

Table 5: unsplittable fbw

\section{Conclusion}

The Flow Deviation algorithm remains a very versatile and prolific tool to solve routing and design problems in networks since the pioneer work of M. Gerla in the seventies. Several variants and algorithmic improvements have been proposed and validated here to take in consideration some bounds on the number of active paths to support the less congested solutions. As the resulting model leads to NPhard problems for most practical situations, heuristic have been first proposed to show how the simplicity of the Flow Deviation updates is very useful to control the number of paths for each commodity. Of course, extreme situations like the unsplittable situation associated with heavy loads are unlikely to yield nice results with these heuristics as the theoretical approximation bounds are known to be quite weak for these hard problems. In these cases, branching procedures and the efficient generation of valid cuts should be added to the basic (FD) iteration to get good solutions. These studies are currently under studies with a limited computational experience on small to medium-size networks.

\section{References}

[1] R. Ahuja, T. Magnanti, and J. Orlin. Network Flows : Theory and Algorithms. Prentice-Hall, Englewood Cliffs, 1993.

[2] G. Baier, E. Köehler, and M. Skutella. On the k-splittable fbw problem. Algorithmica, special issue devoted to ESA 2002, to appear, 2002. 
[3] D. Banerjee and B. Mukherjee. Wavelength-routed optical networks : Linear formulation, resource-budgeting tradeoffs and a reconfiguration study. IEEE/ACM Transactions on Networking, 8(5):598-607, 2000.

[4] D. Bertsekas and R. Gallager. Data networks. Prentice-Hall, Englewood Cliffs, 1987.

[5] D. P. Bertsekas. Nonlinear Programming. Prentice-Hall, Englewood Cliffs, 1995.

[6] D. Bienstock. Potential Function Methods for Approximately Solving Linear Programming Problems : Theory and Practice. Kluwer Publishers, 2003.

[7] D. Bienstock and O. Raskina. Asymptotic analysis of the fbw deviation method for the maximum concurrent fbw problem. Mathematical Programming, 91:479-492, 2002.

[8] J. Burns, T. Ott, A. Krzesinski, and K. Muller. Path selection and bandwidth allocation in mpls networks : a non linear programming approach. Performance Evaluation, 52:133-152, 2003.

[9] M. Frank and P. Wolfe. An algorithm for quadratic programming. Naval Research Logistics Quarterly, 3:95-110, 1956.

[10] L. Fratta, M. Gerla, and L. Kleinrock. The fbw deviation method: an approach to store-and-forward computer-communication network design. Networks, 3, 1973.

[11] N. Garg and J. Könemann. Faster and simpler algorithms for multicommodity fbw and other fractional packing problems. In Proc. 39th Ann. Symp. on Foundations of Computer Science, 1998.

[12] M. D. Grigoriadis and L. G. Khachiyan. Fast approximation schemes for convex programs with many blocks and coupling constraints. SIAM Journal on Optimization, 4(1):86-107, 1994.

[13] J. Kleinberg. Approximation algorithms for disjoint path problems. $\mathrm{PhD}$ thesis, MIT, Cambridge, 1996.

[14] L. B. J. LeBlanc, R. Helgason, and D. Boyce. Improved efficiency of the frankwolfe algorithm for convex network programs. Transportation Sci., 19:445$462,1985$. 
[15] L. B. J. LeBlanc, P. Mahey, and J. Chiffet. Packet routing in telecommunications networks with path and fbw restrictions. INFORMS J. on Computing, 11:188-197, 1999.

[16] L. B. J. LeBlanc, E. Morlock, and W. Pierskalla. An efficient approach to solving the road network equilibrium traffic assignment problem. Trans. Research, 3:309-318, 1975.

[17] F. T. Leighton, F. Makedon, S. Plotkin, C. Stein, E. Tardos, and S. Tragoudas. Fast approximation algorithms for multicommodity fbw problems. Journal of Computer and System Sciences, 50(2):228-243, 1995.

[18] M. Martens and M. Skutella. Flows on few paths : algorithms and lower bounds. In Proc. 12th Annual European Symposium on Algorithms, ESA'04, 2004.

[19] V. Mirrokni, M. Thottan, H. Uzunalioglu, and S. Paul. A simple polynomial time framework for reduced-path decomposition in multi-path routing. In Proc. INFOCOM, 2004.

[20] S. A. Plotkin, D. B. Shmoys, and E. Tardos. Fast approximation algorithms for fractional packing and covering problems. Mathematics of Operations Research, 20(2):257-301, 1995.

[21] F. Shahrokhi and D. W. Matula. The maximum concurrent fbw problem. Journal of the ACM, 37:318-334, 1990.

[22] B. Vatinlen, C. Duhamel, F. Chauvet, and P. Mahey. Minimizing congestion with a bounded number of paths. In Proc. Algotel'03, Banyuls, 2003. 